\title{
Anxiety and Depression Symptoms in Patients with Sleep-Disordered Breathing
}

\author{
Margarita Reyes-Zúñiga ${ }^{1}$, Armando Castorena-Maldonado ${ }^{1}$, \\ José Luis Carrillo-Alduenda ${ }^{1}$, Rogelio Pérez-Padilla ${ }^{1,2}$, \\ Araceli Martínez-Estrada ${ }^{3}$, Leonardo Gómez-Torres ${ }^{4}$ \\ and Luis Torre-Bouscoulet ${ }^{*}, 1,2$
}

\author{
${ }^{1}$ Sleep Medicine Unit, Instituto Nacional de Enfermedades Respiratorias Ismael Cosío Villegas; Mexico City, Mexico \\ ${ }^{2}$ Department of Respiratory Physiology, Instituto Nacional de Enfermedades Respiratorias Ismael Cosío Villegas; \\ Mexico City, Mexico \\ ${ }^{3}$ Universidad La Salle, Faculty of Medicine; Mexico City, Mexico \\ ${ }^{4}$ Hospital Italiano de Buenos Aires; Buenos Aires, Argentina
}

\begin{abstract}
Introduction: Information concerning the frequency of anxiety and depression symptoms in patients with sleepdisordered breathing (SDB) is controversial. Hence, the objectives of this study were to: determine the frequency of anxiety and depression symptoms in patients with SDB; explore whether the frequency of anxiety and depression symptoms rises with increasing parameters of SDB severity; and investigate the factors potentially associated with anxiety and depression.
\end{abstract}

\begin{abstract}
Materials and Methodology: Data was collected from consecutive adult patients referred to the National Institute of Respiratory Diseases' Sleep Medicine Unit from October 2008 to October 2009. SDB diagnoses were established using standard polysomnography or simplified respiratory polygraphy. All patients routinely completed the Hospital Anxiety and Depression Scale (HADS) on their first visit to the Sleep Medicine Unit.

Results: 382 patients with SDB were included. Mean age was $50.8 \pm 13.6$ years, $62 \%$ were male. Anxiety symptoms were acknowledged by 71 patients (18.5\%), while 29 (7.6\%) had depression symptoms, and $116(30.4 \%)$ had symptoms of anxiety plus depression. Patients who reported anxiety and depression symptoms scored higher on the Epworth Sleepiness Scale (ESS, 16 \pm 7$)$ than those with no symptoms $(11 \pm 6)(\mathrm{p}<0.0001)$. Patients with symptoms of anxiety plus depression also had higher BMIs $\left(36 \pm 8 \mathrm{~K} / \mathrm{m}^{2}\right)$ than patients with SDB without anxiety or depression. No other differences were observed, not even with respect to the parameters of the severity of their SDB.
\end{abstract}

Conclusions: Our results support the recommendation that due to their high frequency and potential importance for longterm adherence to CPAP, anxiety and depression symptoms should be included in the routine evaluation of all adults with SDB.

Keywords: Anxiety, depression, sleep-disordered breathing, sleep apnea, mood disorders.

\section{INTRODUCTION}

Chronic diseases are a well-recognized independent risk factor for developing anxiety and depression [1]. In what constitutes a detrimental cycle for patients, anxiety and depression decrease adherence to long-term treatments, thus contributing, at least potentially, to complications or exacerbations of the underlying illness [2].

The prevalence of anxiety and depression has been explored in relation to certain chronic respiratory diseases

\footnotetext{
*Address correspondence to this author at the Department of Respiratory Physiology and Sleep Medicine Unit, Instituto Nacional de Enfermedades Respiratorias Ismael Cosío Villegas; Mexico City, Mexico; Tlalpan 4502, Colonia Sección XVI, Delegación Tlalpan CP 14080, D.F., México; Tel/Fax: (52) (55) 5665-4748; E-mail: luistorreb@gmail.com
}

[3]. For instance, in patients with asthma, chronic obstructive pulmonary disease, or tuberculosis, the frequency of anxiety and depression was greater than that reported for the general population, and was associated with lower quality of life and a reduced rate of adherence to long-term treatment [4]. Nonetheless, limited information is available on the prevalence of anxiety and depression in patients with sleepdisordered breathing (SDB); a condition that, at least theoretically, could negatively affect adherence to continuous positive airway pressure (CPAP) with serious consequences, such as an increased risk of traffic accidents and cardiovascular events $[5,6]$. There is extensive, though still controversial [7, 8], information on the association between symptoms of depression and SBD, but it is possible that the difficulty in documenting that relation lies in the fact that these two afflictions share some of the same symptoms. 
Treating SDB does indeed improve the somatic symptoms of depression, such as sleepiness, fatigue and irritability, but affective impairment persists [9]. Compared to depression, anxiety has been studied much less in patients with SDB, despite the fact that the prevalence of anxiety is higher than that of depression [10]. In an intriguingly complex network of interactions, obesity - a thoroughly documented independent risk factor for SDB [11] - has also been associated, bi-directionally, with anxiety and depression, such that obesity increases the risk for anxiety and depression and vice versa [12]. Moreover, anxiety and depression also appear to be related to insulin resistance, yet another condition that has been shown to have an association with SDB [13].

Despite the complexity of the interactions among these variables (obesity, SDB, insulin resistance, anxiety and depression, adherence to CPAP, etc.), we believe, and therefore hypothesize, that the prevalence of anxiety and depression in patients with SDB is higher than that observed in the general population, and that the severity of SDB is associated with an increase in the frequency of symptoms of anxiety and depression. Thus, the aims of this study were to: (1) report the frequency of symptoms of anxiety and depression in patients with SDB; (2) explore whether the frequency of anxiety and depression symptoms rises with increases in the usual parameters of SDB severity; and, (3) describe potential factors involved in anxiety and depression in patients with SDB. This knowledge will be helpful in determining whether anxiety and depression should be assessed as part of the regular evaluation of all patients with SDB.

\section{MATERIALS AND METHODOLOGY}

The study was carried out at the National Institute of Respiratory Diseases (INER) in Mexico City, and was approved by its Institutional Review Board (no. C12-11). Data were collected retrospectively and appropriate care was taken to protect patients' privacy and identity. The study included data from all consecutive adults with SDB referred to our Sleep Medicine Unit from October 2008 to October 2009. Since October 2008, all adult patients seen at the Institute have routinely completed the Hospital Anxiety and Depression Scale (HADS). When symptoms of anxiety or depression are recognized, the patient is referred for evaluation by a psychiatrist, and pharmacological treatment is begun as needed.

The HADS questionnaire is a self-completed survey made up of 14 Likert-style items; 7 that explore anxiety (HADS-A), and another 7 that probe depression (HADS-D). This scale is widely used in hospital outpatient settings, nonpsychiatric contexts, and as a screening tool for symptoms of anxiety and depression [14]. HADS was administered in Spanish and is a scale that has been previously validated in this language [15]. The cut-off score of 8 was used for both HADS-A and HADS-D [16].

SDB diagnoses were confirmed using one of two methods: standard polysomnography, or simplified respiratory polygraphy. The decision as to which diagnostic test was scheduled depended on the particular sleep medicine specialist involved in each case.
Polysomnography studies were conducted using a 16channel Grass Instrument (model 15LT-PSGA, Gamma 4.1 version, West Warwick, Rhode Island, USA), or a 32channel Alice Sleepware device (version 5, model 1018958, Respironics brand, Murrysville, Pennsylvania, USA). All studies were conducted in accordance with the American Academy of Sleep Medicine's recommendations [17]. The montage utilized included frontal, central and right occipital electroencephalographic derivations: (F4-M1, C4-M1, O2$\mathrm{M} 1$ ), left and right electrooculograms (EOGs), electromyograms (EMGs) of the chin, EMGs of the anterior tibialis muscle to detect leg movements, and an electrocardiogram channel obtained through a unipolar derivation. Airflow was measured simultaneously using an oronasal thermistor and a pressure transducer (Respironics Sleep Sense ${ }^{\mathrm{TM}}$ brand, Murrysville, Pennsylvania, USA), while respiratory movements of the thorax and abdomen were determined through inductance plethysmography (Respiband $^{\mathrm{TM}}$; New York, USA, or bands, Pro tech ${ }^{\mathrm{TM}}$, Murrysville, Pennsylvania, USA). The state of oxygenation was measured by pulse oximetry (Massimo $^{\mathrm{TM}}$ set; Irvine, USA). Studies were evaluated in 30-second epochs in order to identify sleep stages and respiratory events following the guidelines established by the AASM [17]. Simplified respiratory polygraphy was performed in an attended setting at the Sleep Medicine Unit using the Remmers Sleep Recorder (Sagatech, Calgary, Alberta, Canada), which registers oximetry, airflow, snoring, heart rate, leg movement and respiratory effort, in addition to calculating the respiratory disturbance index (RDI) using an automatic algorithm that sequentially scans each recorded oxygen saturation value $(1 \mathrm{~Hz})$. Whenever a drop in a sampled oxygen saturation value is detected, the program assigns an event marker to that reading. Because oxygen saturation values were sampled and recorded at $1 \mathrm{~Hz}$, event markers were separated by no less than one second. When an increase in oxygen saturation was detected, the program determined whether $\geq 3$ consecutive event markers -that is, 3 consecutive decreases in the recorded oxygen saturation readings- had occurred prior to this rise. If this criterion was met and one of the event markers was associated with an oxygen saturation value $>4 \%$ lower than baseline oxygen saturation, then a respiratory disturbance was designated [18]. The quality of the simplified respiratory polygraphy tests was ascertained on the basis of a visual examination performed by an experienced sleep technician. Studies with $<4 \mathrm{~h}$ of recording were not included for analysis $(\mathrm{n}=17)$.

For all cases, SDB was established when either the apnea/hypopnea index or the respiratory disturbance index was $\geq 5$ events/hr. Arterial hypertension was recognized when this diagnosis had been established previously by a physician, or when the patient was on prescribed antihypertensive drugs.

\section{Statistical Analysis}

As most of the variables showed symmetrical distributions, data are presented in the form of means and standard deviations. The categorical variables were described as frequencies and percentages using $95 \%$ confidence intervals $(95 \% \mathrm{CI})$. Group comparisons were carried out, as appropriate, using the Wilcoxon Rank-Sum Test or $X^{2}$. Comparisons among more than two groups were made using 
a one-way ANOVA with a Bonferroni post hoc test or Kruskal Wallis test, and adjusting the $\mathrm{p}$ value for multiple comparisons. In order to explore the factors potentially associated with anxiety or depression symptoms (dependent variables) logistic models were built. The following independent variables were considered: age, gender, body mass index (BMI), neck circumference, the ESS, selfreported sleep time, latency to sleep onset, the apnea/hypopnea index, the respiratory disturbance index, mean nocturnal oxygen saturation $\left(\mathrm{SpO}_{2}\right)$, lowest $\mathrm{SpO}_{2}$, and ingestion of psychiatric drugs. A two-tailed $\mathrm{p}$ value of $<0.05$ or $<0.01$ was considered significant in cases of multiple comparisons. Data analysis was conducted using a commercially available statistical package (Stata, release 10, StataCorp, College Station, TX, USA).

\section{RESULTS}

Three hundred and eighty-two consecutive patients with SDB were included; 171 (44.7\%) were studied using standard polysomnograms, and $211(55.3 \%)$ by simplified respiratory polygraph. Mean age was $50.8 \pm 13.6$ years, and the majority of subjects were male $(62 \%)$. A total of 216 subjects $(56.5 \%)$ were referred for psychiatric evaluation after obtaining HADS scores $\geq 8$. The general characteristics of all patients are shown in Table $\mathbf{1}$.

Table 1. General Characteristics $[n=382]$

\begin{tabular}{|l|c|}
\hline \multicolumn{1}{|c|}{ Parameter } & Value \\
\hline \hline Age, $\mathrm{y}^{*}$ & $50.8[13.6]$ \\
\hline Male; $\mathrm{n}(\%)$ & $236[62]$ \\
\hline Occupation; $\mathrm{n}(\%)$ & \\
Drivers & $30(7.8)$ \\
Professionals & $39(10.2)$ \\
Homemakers & $98(25.6)$ \\
Artisans & $143(37.4)$ \\
Unemployed & $72(18.8)$ \\
\hline Systemic arterial hypertension, $\mathrm{n}$ [\%] & $204(53.4)$ \\
\hline Epworth Sleepiness Scale* & $13[7]$ \\
\hline Psychotropic drugs, $\mathrm{n}(\%)$ & $39(10)$ \\
Antidepressant & $8(2)$ \\
Benzodiazepines & $25(6)$ \\
Antipsychotics & $3(0.8)$ \\
Anticonvulsants & $3(0.8)$ \\
\hline BMI, K/m ${ }^{2 *}$ & $34(7)$ \\
\hline Neck circumference, cm* & $42(4)$ \\
\hline SpO ${ }_{2}$ awake; \%, * & $91.4(4.4)$ \\
\hline Anxiety, $\mathrm{n}$ (\%) & $71(18.5)$ \\
\hline Depression, $\mathrm{n}(\%)$ & $29(7.6)$ \\
\hline Anxiety plus depression, $\mathrm{n}(\%)$ & $116(30.4)$ \\
\hline
\end{tabular}

*Mean $[\mathrm{SD}]$.

BMI body mass index; $\mathrm{SpO}_{2}$, oxygen saturation.

Anxiety symptoms were recognized by 71 patients (18.5\%), while 29 (7.6\%) and $116(30.4 \%)$, respectively, had depression, or symptoms of anxiety plus depression. Thus, a total of 216 subjects $(56.5 \%)$ had psychiatric symptoms related to anxiety, depression, or anxiety plus depression. Table 2 shows the anthropometric and sleep-related parameters according to the occurrence of anxiety or depression symptoms. A lower proportion of females was observed in the no-anxiety or depression symptoms group $(30.7 \%)$ compared to the group with anxiety plus depression symptoms $(50.9 \%)(\mathrm{p}=0.006)$.

Patients with anxiety plus depression symptoms had higher scores on the ESS $(16 \pm 7)$ than those with no symptoms $(11 \pm 6)(\mathrm{p}<0.0001)$. The anxiety plus depression symptoms group had the highest BMI $\left(36 \pm 8 \mathrm{~K} / \mathrm{m}^{2}\right)$. No other significant differences were seen, not even with regards to the severity parameters of their SDB condition (Table 2).

When patients were classified in one of two groups according to anxiety and depression symptoms (i.e., anxiety or depression $v s$ no symptoms, see Table 3), differences were noted with respect to gender (anxiety or depression symptoms were more often associated with women), the ESS (anxiety or depression symptoms were associated with increased sleepiness), BMI (anxiety or depression symptoms were associated with higher BMI), and use of psychiatric drugs (symptoms were associated with medication). SDB severity indices were similar in the two groups.

Table 4 shows the raw odds ratios for anxiety, depression, and anxiety plus depression symptoms. Age was identified as a protective factor for anxiety (OR 0.98, 95\% CI 0.96-0.99, $\mathrm{p}=0.01$ ), while a weak, but significant, association was seen between the apnea/hypopnea index and the presence of anxiety plus depression symptoms (OR 1.006, 95\% CI 1-1.01, $\mathrm{p}=0.03)$. As expected, a relation was demonstrated between psychiatric drugs and anxiety symptoms. The odds ratios adjusted for potential confounders are shown in Table $\mathbf{5}$.

\section{DISCUSSION}

In Mexico, the prevalence of anxiety and affective disorders in the general population is 14.3 and $9.1 \%$, respectively [19]. It has been estimated that anxiety disorders affect $18.1 \%$ of the adult population of the United States, while mood disorders afflict 9.5\% [18]. Compared to local population data, the frequency of symptoms of anxiety or depression documented in this study of patients with SDB shows a substantially higher figure (57\%). Our study indicates that women have a higher incidence of symptoms of anxiety or depression than men, and that the lower the age the greater the symptomatology. These findings concur with observations made of the general population, where feminine gender and lower age are risk factors for suffering symptoms of anxiety; whereas symptoms of depression are more frequent in the adult age group [20].

The association between SDB and anxiety and depression has been explored in previous studies $[6,7,21]$, but with inconsistent results. In a longitudinal study of 1, 408 subjects using adjusted models, Peppard et al. identified SDB as a risk factor for developing depression, and isolated a doseresponse effect which revealed that the probability of developing depression increased 1.6-fold (95\% CI, 1.2-2.1) in participants with minimal SDB; 2.0 -fold (95\% CI, 1.4-2.9) 
Table 2. Anthropometric and Sleep Parameters According to Anxiety or Depression

\begin{tabular}{|c|c|c|c|c|c|}
\hline & $\begin{array}{c}\text { Anxiety } \\
\mathrm{n}=71\end{array}$ & $\begin{array}{l}\text { Depression } \\
\quad \mathbf{n}=\mathbf{2 9}\end{array}$ & $\begin{array}{l}\text { Anxiety Plus Depression } \\
\qquad \mathrm{n}=116\end{array}$ & $\begin{array}{l}\text { No Anxiety, } \\
\text { No Depression n=166 }\end{array}$ & P Value \\
\hline Age, y;* & $51[13]$ & $54[16]$ & $48[13]$ & $52[13]$ & 0.047 \\
\hline Female; n (\%) & $27(38)$ & $9(31)$ & $59(50.9)$ & $51(30.7)$ & 0.006 \\
\hline Diagnosis of arterial hypertension, $\mathrm{n}(\%)^{* *}$ & $42(59.1)$ & $16(55.1)$ & $59(50.8)$ & $87(52.4)$ & 0.71 \\
\hline Epworth Sleepiness scale, ${ }^{*}$ & $12[7]$ & $15[6]$ & $16[7]$ & $11[6]$ & $<0.0001$ \\
\hline Self-reported sleep time, hours;* & $7[1.5]$ & $8[1.4]$ & $6.8[1.7]$ & $7[1.4]$ & 0.08 \\
\hline Self-reported sleep latency, minutes;* & $30[31]$ & $23[25]$ & 24 [27] & $20[21]$ & 0.18 \\
\hline $\mathrm{BMI}, \mathrm{K} / \mathrm{m}^{2} ; *$ & $34[6]$ & $32[6]$ & $36[8]$ & $33[6]$ & 0.0005 \\
\hline Neck circumference, cm;* & $42[5]$ & $41[4]$ & $43[5]$ & $43[4]$ & 0.49 \\
\hline Mean sleep $\mathrm{SpO}_{2,} \%$; & $84[9]$ & $85[7]$ & $82[11]$ & $83[8]$ & 0.20 \\
\hline Minimum sleep $\mathrm{SpO}_{2}, \%$;* & $65[15]$ & $64[17]$ & $61[18]$ & $60[16]$ & 0.22 \\
\hline AHI or RDI, events/hr;* & $51[38]$ & $48[35]$ & $60[42]$ & $52[34]$ & 0.18 \\
\hline Use of Psychotropic drugs, n (\%) & $11(15.5)$ & $3(10.3)$ & $15(12.9)$ & $10(6)$ & 0.09 \\
\hline
\end{tabular}

*Mean $[\mathrm{SD}] ; * *$ Previous diagnosis of high blood pressure, or currently taking antihypertensive drugs.

BMI, body mass index; AHI, apnea/hypopnea index; SpO2, oxygen saturation; RDI, respiratory disturbances index.

By gender, the percentage was different in the anxiety plus depression group $v s$ all others. For the Epworth Scale, significant differences were observed between the no-anxiety/nodepression group $v s$ the anxiety-plus-depression group, as well as between the anxiety group $v s$ the anxiety-plus-depression group. For BMI, significant differences were noted between the depression group $v s$ the anxiety-plus-depression group, as well as in the anxiety-plus-depression group $v s$ the no-anxiety/no-depression group.

Table 3. Anthropometric and Sleep Parameters According to the Presence or Absence of Symptoms of Anxiety or Depression

\begin{tabular}{|c|c|c|c|}
\hline & $\begin{array}{l}\text { SDB and Anxiety or Depression } \\
n=216\end{array}$ & $\begin{array}{l}\text { SDB and No Anxiety or Depression } \\
n=166\end{array}$ & P Value \\
\hline Age, $y ; *$ & $49.7[13.6]$ & $52.3[13.3]$ & 0.05 \\
\hline Male; $n[\%]$ & $95[44]$ & $115[70]$ & 0.008 \\
\hline Diagnosis of arterial hypertension; $\mathrm{n}[\%]^{* *}$ & $117[54.1]$ & $87[52.4]$ & 0.73 \\
\hline Epworth Sleepiness Scale, * & $14.5[7]$ & $11[6.4]$ & $<0.001$ \\
\hline Self-reported sleep latency, minutes; ${ }^{*}$ & $26[28]$ & $20[21]$ & 0.09 \\
\hline BMI, $\mathrm{K} / \mathrm{m}^{2} ; *$ & $34.8[7.5]$ & $33.1[6.3]$ & 0.02 \\
\hline Neck circumference, $\mathrm{cm} ; *$ & $42.3[4.5]$ & $42.6[4]$ & 0.51 \\
\hline Mean sleep $\mathrm{SpO}_{2,} \% ; *$ & $83.3[9.8]$ & $83.4[8]$ & 0.9 \\
\hline Use of psychotropic drugs, $n$ [\%] & $29[13.4]$ & $10[6.0]$ & 0.01 \\
\hline
\end{tabular}

*Mean [SD]; ** Previous diagnosis of high blood pressure, or currently taking antihypertensive drugs.

SDB, sleep-disordered breathing; BMI, body mass index; AHI, apnea/hypopnea index; SpO2, oxygen saturation; RDI, respiratory disturbances index.

in subjects with mild SDB; and 2.6-fold (95\% CI, 1.7-3.9) in patients with moderate-to-severe SDB [6]. In stark contrast to those findings, Macey et al. failed to document any association whatsoever between the severity of SDB and the scale of the symptoms of anxiety or depression in patients with no co-morbid states and recent diagnoses [7]. These data suggest that mechanisms beyond the number and frequency of hypoxic events and arousals that occur with apneas contribute to the adverse health effects of SDB [22]. In this context, anxiety has been explored less thoroughly than depression. Moreover, there seems to be no association between sleepiness and symptoms of depression, on the one hand, and SDB, on the other. Our results reiterate the absence of any relation between the severity of SDB and the presence of symptoms of depression and anxiety; however, the symptoms of anxiety plus depression as measured by the HADS are more pronounced in our sample than those obtained for the population as a whole. Symptoms of depression and anxiety may form part of the symptomatic complex of SDB, due to the damage that intermittent hypoxemia causes in the limbic structures, the anterior cingulate, the insula, the amygdala and the hippocampus, 
Table 4. Simple Odds Ratios According to Anxiety or Depression Status

\begin{tabular}{|l|c|c|c|c|c|c|}
\hline \multirow{2}{*}{} & \multicolumn{2}{|c|}{ Anxiety } & \multicolumn{2}{c|}{ Depression } & \multicolumn{2}{c|}{ Anxiety Plus Depression } \\
\cline { 2 - 7 } & OR 95\%CI & p & OR 95\%CI & p & OR 95\%CI & p \\
\hline \hline Age & $0.98[0.96-0.99]$ & 0.01 & $0.98(0.97-1.00)[6]$ & 0.07 & $0.97[0.96-0.99]$ & 0.01 \\
\hline Male & $0.52[0.34-0.79]$ & 0.002 & $0.55(0.36-0.84)[6]$ & 0.007 & $0.46[0.30-0.73]$ & 0.001 \\
\hline BMI & $1.05[1.01-1.08]$ & 0.001 & $1.04(1.01-1.07)[6]$ & 0.007 & $1.06[1.03-1.096]$ & $<0.001$ \\
\hline NC & $1[0.95-1.05]$ & 0.17 & $0.98[0.93-1.03]$ & 0.51 & $1[0.95-1.05]$ & 0.84 \\
\hline Epworth Sleepiness Scale & $1.06[1.03-1.09]$ & $<0.0001$ & $1.10[1.06-1.14]$ & $<0.001$ & $1.10[1.06-1.14]$ & $<0.001$ \\
\hline Self-reported sleep time & $0.87[0.76-1.00]$ & 0.06 & $0.98[0.85-1.13]$ & 0.86 & $0.89[0.77-1.04]$ & 0.15 \\
\hline Self-reported sleep latency & $1[0.99-1.01]$ & 0.08 & $1[0.99-1.01]$ & 0.89 & $1[0.99-1.01]$ & 0.81 \\
\hline AHI & $1[0.99-1.00]$ & 0.17 & $1[0.99-1.00]$ & 0.12 & $1.006[1.002-1.01]$ & 0.03 \\
\hline Mean sleep SpO $\mathrm{S}_{2}$ & $0.99[0.96-1.01]$ & 0.42 & $0.98[0.96-1.01]$ & 0.35 & $0.97[0.95-1]$ & 0.08 \\
\hline Minimum sleep SpO & $1[0.99-1.01]$ & 0.28 & $1[0.98-1.01]$ & 0.94 & $0.99[0.98-1.01]$ & 0.73 \\
\hline Arterial hypertension & $1.04[0.70-1.56]$ & 0.81 & $0.89[0.59-1.35]$ & 0.60 & $0.86[0.55-1.33]$ & 0.51 \\
\hline Psychotropic drugs & $1.45[1.02-2.07]$ & 0.03 & $1.04[0.75-1.45]$ & 0.79 & $1.49[0.75-2.9]$ & 0.24 \\
\hline
\end{tabular}

$\mathrm{BMI}$, body mass index; $\mathrm{NC}$, neck circumference; $\mathrm{AHI}$, apnea/hypopnea index; $\mathrm{SpO}$, oxygen saturation.

Table 5. Adjusted Odds Ratios According to Anxiety or Depression Status; Significant Associations are Shown

\begin{tabular}{|c|c|c|c|c|c|c|}
\hline & \multicolumn{2}{|c|}{ Anxiety } & \multicolumn{2}{|c|}{ Depression } & \multicolumn{2}{|c|}{ Anxiety Plus Depression } \\
\hline Male & $0.26[0.14-0.47]$ & $<0.001$ & $0.39[0.24-0.63]$ & $<0.0001$ & $0.36[0.21-0.61]$ & $<0.001$ \\
\hline Epworth Sleepiness Scale & $1.12[1.07-1.17]$ & $<0.001$ & $1.11[1.07-1.15]$ & $<0.0001$ & $1.11[1.07-1.15]$ & $<0.001$ \\
\hline Self-reported sleep time & $0.81[0.66-0.99]$ & 0.042 & - & - & - & - \\
\hline BMI & -- & - & - & - & $1.04[1.01-1.08]$ & 0.009 \\
\hline
\end{tabular}

BMI, body mass index.

The independent variables included in the models are mentioned in the section "Statistical analysis".

and not necessarily to a primary mood disorder [23]. The subjects that manifested more symptoms associated with anxiety and affect experience greater daytime sleepiness, likely a manifestation of the more severe damage to structures vulnerable to intermittent hypoxemia and sleep fragmentation.

Our study found that individuals with SDB who experience symptoms of anxiety plus depression have higher BMIs than SDB patients who do not experience those symptoms; results that agree with those described by Luppino et al., who documented that obesity increases the risk of depression [24]. The activity of the hypothalamushypophysis-suprarenal axis is deregulated in obesity as "functional hypercortisolism" [25]. There is a bidirectional communication between the hypothalamus-hypophysissuprarenal axis and the adipose tissue, and the deregulation of this axis is the most common biological finding in depression [26]. Obesity and depression have a common denominator in a chronic inflammatory condition, the mediators of which may be the underlying cause of the symptoms of depression [27]. Alterations in the adipokines share effects in obesity and cases of mood alterations. Rats exposed to chronic stress show low levels of leptin and depression-like behaviors that diminish when that substance is administered [28]. Leptin participates in the immune response by increasing the production of IL- 6 and TNF- $\alpha$; while, as previous research has demonstrated, leptin insufficiency, or leptin resistance, may exacerbate an individual's vulnerability to depression [29]. On the other hand, hypoadiponectinemia has been suggested as a mediating factor in the association between obesity and mood disorders. In an interesting study, Leo et al. showed that patients with major depression displayed lower adiponectin plasma levels compared to controls, and that adiponectin significantly correlated with the severity of depression [30].

The elevated frequency of symptoms of anxiety plus depression in patients with SDB may well have various implications from the practical perspective. For example, in relation to treatment, Wells et al. demonstrated that there is no correlation between the symptoms of depression and mean use of CPAP per night [31]; while Harris et al. 
concluded that pharmacological treatment of depression might improve acceptance of CPAP therapy, reduce sleepiness and fatigue, and thus improve quality of life [32]. On the other hand, in a study of a small number of patients with SDB, El-Sherbini reported depressive symptoms in $57 \%$ of subjects referred with CPAP treatment [33]. At this time, there is no published information on the acceptance of, and adherence to, CPAP treatment as they relate to symptoms of anxiety. Controlled clinical studies are needed to elucidate whether treatment for anxiety prior to beginning CPAP might improve long-term adherence to this therapy.

Our study has limitations, such as the fact that no control group was involved, and that not all patients were tested by polysomnography; conditions that preclude any exploration as to whether sleep architecture is associated with the symptoms of anxiety or depression in patients with SDB. SDB diagnoses were confirmed using standard polysomnography or simplified respiratory polygraphy, and $\mathrm{AHI}$ and RDI were used interchangeably. Though it is clear that it is not recommendable to use AHI and RDI in this way, the agreement between the AHI and RDI results in adults with suspected obstructive sleep apnea syndrome in Mexico City was reported as high, with an intra-class correlation coefficient of $0.89 \pm 0.03$ (IC 95\%, 0.83-0.96) [34]. Another limitation is that the HADS and ESS are selfreported scales that could over or under-report symptoms. Although we were not able to perform an objective measurement of sleepiness, ESS is a well-accepted tool for assessing this particular symptom in patients with SDB [35]. In SDB patients, obesity is usually a difficult-to-adjust-for confounder. Indeed, patients with anxiety or depressive symptoms had a higher BMI compared to those with no anxiety or depressive symptoms; a finding which suggests that obesity could be an independent risk factor. We believe that no well-known, complex biological interactions among obesity, hormonal abnormalities, intermittent hypoxemia and inflammatory status leading anxiety or depression in SDB patients exist. One strength of our study is that it did include subjects with diverse degrees of severity of SDB, and that the scale used to measure the symptoms of anxiety and depression (HADS) can be used as an efficacious, userfriendly screening test to evaluate symptoms of those conditions, and to exclude such items as somatic symptoms like fatigue or sleepiness that may be more closely associated with SDB than mood alterations. While HADS certainly cannot replace assessment by a mental health specialist, it can alert physicians as to treatment requirements, or the need to refer patients to other levels of medical attention. Also, HADS has been utilized in patients with COPD, where it has shown that symptoms of anxiety and depression are associated with higher mortality, longer hospitalizations, persistence of habitual smoking, and a worsening of physical and social functionality [36]. Finally, we recognize that our group of patients is highly selective and may therefore not reflect the general population.

In conclusion, due to the high frequency of affective and anxiety disorders that this study demonstrated, we recommend that the presence of affective symptoms be evaluated as part of the routine assessment of SDB patients.

\section{CONFLICT OF INTEREST}

The authors confirm that this article content has no conflicts of interest.

\section{ACKNOWLEDGEMENTS}

The authors would like to thank Mr. Paul C. Kersey (Language Laboratory, El Colegio de Michoacán) for correcting language use.

\section{REFERENCES}

[1] Screening for depression: recommendations and rationale. Ann Intern Med 2002; 136: 760-4.

[2] Dirmaier J, Watzke B, Koch U, et al. Diabetes in primary care: prospective associations between depression, nonadherence and glycemic control. Psychother Psychosom 2010; 79: 172-8.

[3] Kunik, M, Roundy K, Veazy C, et al. Surprisingly high relevance of anxiety and depression in chronic breathing disorders. Chest 2005; 127: 1205-12.

[4] Moussas G, Tselebis A, Karkanias A, et al. A comparative study of anxiety and depression in patients with bronchial asthma, chronic obstructive pulmonary disease and tuberculosis in a general hospital of chest diseases. Ann Gen Psychiatry 2008; 7: 7.

[5] Caples SM, Garcia-Touchard A, Somers VK. Sleep-disordered breathing and cardiovascular risk. Sleep 2007; 30: 291-303.

[6] Barbé F, Pericas J, Munoz A, Findley L, Anto JM, Agusti AG. Automobile accidents in patients with sleep apnea syndrome. An epidemiological and mechanistic study. Am J Respir Crit Care Med 1998; 158: 18-22.

[7] Peppard PE, Szklo-Coxe M, Hla KM, Young T. Longitudinal association of sleep-related breathing disorder and depression. Arch Intern Med 2006; 166: 1709-15

[8] Macey PM, Woo MA, Kumar R, Cross RL, Harper RM. Relationship between obstructive sleep apnea severity and sleep, depression and anxiety symptoms in newly-diagnosed patients. PLoS One 2010; 5: e10211.

[9] El-Ad B, Lavie P. Effect of sleep apnea on cognition and mood. Int Rev Psychiatry 2005; 17: 277-82.

[10] Andrews, JG. Oei T. The roles of depression and anxiety in the understanding and treatment of Obstructive Sleep Apnea Syndrome. Clin Psychol Rev 2004; 24: 1031-49.

[11] Young T, Shahar E, Nieto FJ, et al. Predictors of sleep-disordered breathing in community-dwelling adults: the Sleep Heart Health Study. Arch Intern Med 2002; 162: 893-900.

[12] Anderson SE, Cohen P, Naumova EN, Must A. Association of depression and anxiety disorders with weight change in a prospective community-based study of children followed up into adulthood. Arch Pediatr Adolesc Med 2006; 160: 285-91.

[13] Pearson S, Schmidt M, Patton G, et al. Depression and insulin resistance: cross-sectional associations in young adults. Diabetes Care 2010; 33: 1128-33.

[14] Zigmond AS, Snaith RP. The hospital anxiety and depression scale. Acta Psychiatr Scand 1983; 67: 361-70.

[15] Quintana JM, Padierna A, Esteban C, Arostegui I, Bilbao A, Ruiz I. Evaluation of the psychometric characteristics of the Spanish version of the hospital anxiety and depression scale. Acta Psychiatr Scand 2003; 107: 216-21.

[16] Bjelland I, Dahl AA, Tangen HT, Necklmann D. The validity of the Hospital Anxiety and Depression Scale an updated literature review. J Psychosom Res 2002; 52: 69-77.

[17] Iber C, Ancoli-Israel S, Chesson A, Quan SF. The American Academy of Sleep Medicine Manual for the Scoring of Sleep and Associated Events. Rules, terminology and technical specifications. Westchester, Illinois: American Academy of Sleep Medicine 2007.

[18] Issa FG, Morrison D, HadjukE, Iyer A, Feroah T, Remmers JE. Digital monitoring of sleep-disordered breathing using snoring sound and arterial oxygen saturation. Am Rev Respir Dis 1993; 148(4 Pt 1): 1023-9.

[19] Medina-Mora ME, Borges G, Benjet C, Lara C, Berglund P. Psychiatric disorders in Mexico: lifetime prevalence in a nationally representative sample. Br J Psychiatry 2007; 190: 521-8. 
[20] Kessler RC, Angermeyer M, Anthony JC, et al. Lifetime prevalence and age-of-onset distributions of mental disorders in the World Health Organization's World Mental Health Survey Initiative. World Psychiatry 2007; 6: 168-76.

[21] Ohayon MM. The effect of breathing related sleep disorders on mood disturbances in the general population. J Clin Psychiatry 2003; 64: 1195-200.

[22] Sforza E, De Saint HZ, Pelissolo A, Rochat T, Ibanez V. Personality, anxiety and mood traits in patients with sleep-related breathing disorders: effect of reduced daytime alertness. Sleep Med 2002; 3: 139-45.

[23] Macey PM, Kumar R, Woo MA, Valladares EM, Yan-Go FL, Harper RM. Brain structural changes in obstructive sleep apnea. Sleep 2008; 31: 967-77.

[24] Luppino FS, de Wit LM, Bouvy PF, et al. Overweight, obesity, and depression: a systematic review and meta-analysis of longitudinal studies. Arch Gen Psychiatry 2010; 67: 220-9.

[25] Bjorntorp P. Visceral obesity: a "civilization syndrome". Obesity Res 1993; 1: 206-22.

[26] Bornstein SR, Schuppenies A, Wong ML, Licinio J. Approaching the shared biology of obesity and depression: the stress axis as the locus of gene-environment interactions. Mol Psychiatry 2006; 11: 892-902.

[27] Korenblum W, Barthel A, Licinio J, et al. Elevated cortisol levels and increased rates of diabetes and mood symptoms in Soviet Union-born Jewish immigrants to Germany. Mol Psychiatry 2005; 10: 974-5.

[28] Lu XY. The leptin hypothesis of depression: a potential link between mood disorders and obesity? Curr Opin Pharmacol 2007; 7: 648-52.
[29] Lu XY, Kim CS, Frazer A, Zhang W. Leptin: a potential novel antidepressant. Proc Natl Acad Sci USA 2006; 103: 1593-8.

[30] Leo R, Di Lorenzo G, Tesauro M, et al. Decreased plasma adiponectin concentration in major depression. Neurosci Lett 2006; 407: 211-3.

[31] Wells RD, Freedland KE, Carney RM, Duntley SP, Stepanski EJ. Adherence, reports of benefits, and depression among patients treated with continuous positive airway pressure. Psychosom Med 2007; 69: 449-54.

[32] Harris M, Glozier N, Ratnavadivel R, Grunstein R. Obstructive sleep apnea and depression. Sleep Med Rev 2009; 13: 437-44.

[33] El-Sherbini AM, Bediwy AS, El-Mitwalli A. Association between obstructive sleep apnea and depression and the effect of continuous positive airway pressure (CPAP) treatment. Neuropsychiatr Dis Treat 2011; 7: 715-21

[34] Torre-Bouscoulet L, Castorena-Maldonado A, Baños-Flores R Vázquez-García JC, Meza-Vargas MS, Perez-Padilla R. Agreement between oxygen desaturation index and apnea-hypopnea index in adults with suspected obstructive sleep apnea at an altitude of 2240 m. Arch Bronconeumol 2007; 43: 649-54

[35] Kushida C, Littner M, Morgenthaler T, et al. Practice parameters for the indications for Polysomnography and related procedures: an update for 2005. Sleep 2005; 28: 505-19.

[36] Ng TP, Niti M, Tan WC, Cao Z, Ong KC, Eng P. Depressive symptoms and chronic obstructive pulmonary disease: effect on mortality, hospital readmission, symptom burden, functional status, and quality of life. Arch Intern Med 2007; 167: 60-7.

(C) Reyes-Zúñiga et al.; Licensee Bentham Open.

This is an open access article licensed under the terms of the Creative Commons Attribution Non-Commercial License (http://creativecommons.org/licenses/by-nc/3.0/) which permits unrestricted, non-commercial use, distribution and reproduction in any medium, provided the work is properly cited. 